\title{
Mortalidade perinatal e infantil em Pelotas, Rio Grande do Sul: nossas estatísticas são confiáveis?
}

\author{
* Universidade Católica de \\ Pelotas, $R S$. \\ ** Universidade Federal de \\ Pelotas, RS. \\ *** Projeto "Estudo longitudinal \\ das crianças nascidas em $1982 \mathrm{em}$
} Pelotas, $R S$.

\author{
Fernando C. Barros ${ }^{*}{ }^{* * *}$ \\ Cesar G. Victoria ${ }^{* *}, * *$ \\ Ana Maria Borges Teixeira ${ }^{* * *}$ \\ Miguel Puerto Filho**
}

Uma coorte de 6.011 crianças vem sendo acompanhada desde o nascimento em Pelotas, $R S$. A última avaliação foi realizada quando as crianças tinham 20 meses, em média. Este acompanhamento foi feito através de um censo da cidade, quando todas as 68.600 residências foram visitadas, tendo sido localizadas $87,3 \%$ das crianças da coorte.

Concomitantemente, todos os óbitos foram monitorizados através de visitas domiciliares, revisão periódica de atestados de óbito na Secretaria da Saúde e revisão de prontuários hospitalares.

Com o uso dessa metodologia, foi possivel detectar $42,1 \%$ de sub-registros de mortes perinatais, com $47,8 \%$ para óbitos fetais. Com relação à mortalidade infantil, a proporção de sub-registros foi de $24 \%$.

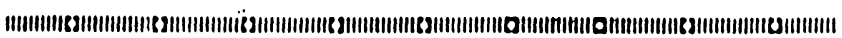

\section{INTRODUÇÃO}

Vários indicadores de saúde têm sido utilizados internacionalmente como instrumento para a monitorização da qualidade de vida de populações. Entre estes indicadores, os coeficientes de mortalidade infantil são, certamente, os mais amplamente empregados na avaliação do desenvolvimento de distintas regiões.

Os coeficientes de mortalidade perinatal também vêm assumindo um papel de destaque como indicadores de saúde, principalmente em áreas submetidas a rápidas transformações sociais. Nestes locais, o desenvolvimento social faz com que os coeficientes de mortalidade infantil caiam, às custas, basicamente, da redução de mortes pós-neonatais, que são mais suscetíveis a ações preventivas, como saneamento básico e imunizações. Como conseqüência, à medida que uma 
região aumenta seu nível de desenvolvimento, maior será a importância relativa das mortes ocorridas na primeira semana ou no primeiro mês de vida, e, em alguns países, o número de mortes ocorridas no período perinatal excede aquele ocorrido nos 30 anos subseqüentes ${ }^{7}$.

Também a distribuição do peso ao nascer tem sido cada vez mais utilizada como um indicador, não só de saúde, mas de desenvolvimento global. Alguns autores sugerem que a incidência de recém-nascidos de baixo peso $(<2.500 \mathrm{~g})$ é mais importante do que indicadores econômicos classicamente utilizados, como o produto interno bruto (PIB) per capita, pois esses não são capazes de medir a distribuição da riqueza dentro de uma sociedade ${ }^{3}$. De fato, os coeficientes de mortalidade perinatal e a incidência de baixo peso ao nascer refletem uma complexa interação de fatores sociais, econômicos, políticos, demográficos e de atenção à saúde, e portanto podem auxiliar não somente na avaliação dos serviços de atenção à saúde, mas também na comparação da qualidade de vida de diferentes grupos sociais dentro de uma mesma população.

Um problema que impede a plena utilização desses indicadores em países em desenvolvimento é o sub-registro de nascimentos e de óbitos perinatais e infantis, que faz com que muitas estatísticas oficiais não sejam dignas de confiança.

No estado do Rio Grande do Sul, reduções sucessivas nos coeficientes de mortalidade infantil têm sido descritas nos últimos anos, e as causas perinatais de óbitos infantis têm sido cada vez mais importantes, tendo aumentado de $22,2 \%$, em 1970 , para $42,6 \%$, em $1981^{5}$. Entretanto, investigações que possam testar a qualidade destes registros oficiais, mediante a monitorização de eventos vitais através de outras fontes de informações, têm sido raramente realizadas. Um estudo levado a efeito em 1974 encontrou $31 \%$ de sub-registro para óbitos gerais ${ }^{6}$, mas acredita-se que a qualidade das estatísticas vitais desse Estado tenha melhorado nos últimos anos, possibilitando a utilização dos indicadores citados como instrumentos de avaliação de saúde pública.

O projeto de investigação "Estudo longitudinal das crianças nascidas em 1982 em Pelotas" possibilitou-nos a avaliação dos registros oficiais de nascimento e óbitos perinatais e infantis, e nossos achados são aqui apresentados.

No que diz respeito à cidade, é importante salientar que Pelotas tem algumas características que favorecem este tipo de estudo: é um município de porte médio (260.000 habitantes em 1980), com população predominantemente urbana $(80 \%)$ e com uma vasta rede de serviços de saúde. A cidade possui duas Faculdades de Medicina, 1 médico para 430 
*BARROS, F.C. et alii. The risk approach in perinatal health care: does it apply to Third World cities? World Health Forum (no prelo) habitantes, e é também sede de uma Delegacia Regional de Saúde.

\section{METODOLOGIA}

Detalhes da metodologia empregada já foram previamente descritos ${ }^{1,2,10 *}$. O estudo dividiu-se nas seguintes etapas (ilustradas na Figura 1):

\section{ESTUDO PERINATAL}

Durante o ano de 1982, todos os nascimentos hospitalares ocorridos na cidade foram estudados por uma equipe formada por médicos e estudantes de Medicina previamente treinados. A investigação realizou-se em três níveis:

Estudo hospitalar: duas vezes por dia os hospitais com maternidade da cidade eram visitados, e informações sobre todos os nascimentos eram colhidas através de uma entrevista com as mães, utilizando-se um questionário padronizado e precodificado. Os recém-nascidos foram pesados (inclusive os natimortos), e suas mães foram pesadas e medidas. Especial atenção foi dada às condições de saúde dos recém-nascidos, e aqueles que se encontravam doentes foram acompanhados cuidadosamente pela equipe. No caso de óbito, procurou-se determinar as possíveis causas através do estudo dos prontuários, e os médicos responsáveis foram contactados sempre que se julgou necessário.

Foram incluídos no estudo recém-nascidos que satisfizessem os seguin tes critérios:

a) para nascidos vivos: peso igual ou superior a $500 \mathrm{~g} \mathrm{e}$ qualquer sinal de vida ao nascer;

b) para natimortos: idade gestacional igual ou superior a 28 semanas ou, em caso de idade gestacional desconhecida, peso ao nascer igual ou superior a $1.000 \mathrm{~g}$.

Visitas domiciliares: Como o período perinatal finda no sétimo dia de vida, e as altas hospitalares em Pelotas costumam ser muito precoces (freqüentemente nas primeiras 24 horas após o parto), tornou-se necessário visitar em casa, após a primeira semana, uma amostra representativa da população estudada. Foram estudados $18,5 \%$ dos recém-nascidos de área urbana ( $15 \%$ do total), para levantar a situação de saúde da criança e de sua mãe.

Revisão de atestados de óbito e certidões de nascimento. Mensalmente, a partir de janeiro de 1982, todos os atestados de óbito e certidões de nascimento de crianças nascidas em 1982 são revisados na Delegacia de Saúde de Pelotas. Através do nome da mãe, endereço, data de nascimento e hospital de nascimento, tem sido possível ligar as informações dos atestados de nascimento e de óbito com o questio. nário hospitalar. Os nascimentos certificados pela família 
como domiciliares foram comparados com os questionários hospitalares, para se saber se tinham, efetivamente, ocorrido em casa. $\mathrm{O}$ número de nascimentos que os hospitais informaram mensalmente à Delegacia de Saúde também tem sido alvo de avaliação.

\section{ACOMPANHAMENTO DAS \\ CRIANÇAS NASCIDAS EM 1982}

Primeiro acompanhamento. Tentou-se localizar, no inicio de 1983, através dos endereços fornecidos no hospital, as crianças nascidas entre janeiro e abril de 1982, e residentes em área urbana, que estavam então com idade média de 12 meses. Foi possível encontrar $81 \%$ destas crianças, e, em cada caso, uma avaliação antropométrica foi feita e um questionário foi completado. Quando um óbito era detectado, procurava-se localizar o atestado e o prontuário hospitalar (se o óbito havia ocorrido em hospital).

Segundo acompanhamento. Esta fase do estudo inclui a visita, no início de 1984 , a todos os domicílios urbanos de Pelotas (cerca de 68.600), para tentar localizar todas as crianças nascidas em 1982. Visitou-se, também, uma amostra de $20 \%$ de crianças residentes em áreas rurais do município. Em cada domicílio, perguntou-se sobre crianças nascidas em 1982, mesmo sobre as que houvessem falecido. As crianças sobreviventes, então com 12 a 27 meses de idade, foram novamente pesadas e medidas, e foi colhido um grande número de informações sobre morbidade, desenvolvimento psicomotor, utilização dos serviços de saúde, etc. Quando um óbito era detectado, novamente procurava-se pelo atestado correspondente, e uma parte especial do questionário era preenchida com a família.

\section{MONITORIZAÇÃO DOS \\ ÓBITOS DAS CRIANÇAS NASCIDAS EM 1982}

Paralelamente aos acompanhamentos domiciliares, a mortalidade destas crianças tem sido monitorizada através da revisão dos atestados de óbito que chegam à Secretaria da Saúde e do Meio Ambiente do Estado e aos cartórios de registro de Pelotas.

Outra fonte de informações é a revisão periódica dos prontuários hospitalares de todas as crianças falecidas na cidade, através de contatos semanais com todos os hospitais. Sempre que um óbito é descoberto através do atestado ou do prontuário hospitalar, as informações neles contidas são conectadas àquelas dos questionários perinatais e dos acompanhamentos domiciliares, através do nome da criança, nome da mãe, e data e local de nascimento. 


\section{ANÁLISE DE DADOS}

Todas as informações coletadas durante as diferentes fases da pesquisa foram digitadas em fita magnética e analisadas em computador, usando-se para isso o pacote SPSS ${ }^{4}$.

\section{RESULTADOS}

\section{REGISTRO DE NASCIMENTOS}

De 1 ? de janeiro a 31 de dezembro de 1982, 7.398 crianças nasceram nos hospitais de Pelotas. A equipe da pesquisa conseguiu avaliar 7.392 recém-nascidos, e nos 6 casos restantes as famílias deixaram o hospital antes de serem entrevistadas.

Revisando as notificações de nascimentos encaminhadas à Secretaria da Saúde e do Meio Ambiente do Estado pelos hospitais, encontramos somente 6.836 recém-nascidos, o que nos dá uma taxa de sub-registro de $7,8 \%$.

Em 1982, encontramos, para a cidade de Pelotas, 358 recém-nascidos registrados pelas famílias como tendo nascido em casa, representando $5 \%$ do total de nascimentos. Através da data de nascimento e do nome da mãe, descobrimos que 239 destas crianças tinham, na realidade, nascido em hospitais da cidade. Os restantes 119 nascimentos registrados como domiciliares podem ter realmente ocorrido em casa, mas muitas destas crianças podem ter nascido em hospitais, tendo sido registradas em casa com data de nascimento alterada. Outra possibilidade é que o nome fornecido pela mãe no hospital tenha sido diferente daquele utilizado durante o registro, o que poderia impossibilitar a detecção do parto hospitalar. Encontramos muitas mães que foram internadas no hospital utilizando carteiras do INAMPS pertencentes a outras pessoas.

Se as 119 crianças que não conseguimos localizar nos questionários hospitalares realmente nasceram em casa, teremos uma taxa de partos domiciliares de 1,6\%. Entretanto, esta cifra parece ser demasiado elevada, pois durante o censo da cidade, que realizamos por ocasião da segunda visita de acompanhamento, encontramos menos de $1,0 \%$ de nascimentos domiciliares em área urbana ${ }^{10}$. Convém salientar que, durante o referido censo, todas as crianças nascidas em 1982 foram examinadas, mesmo as que não pertenciam à nossa coorte de recém-nascidos.

\section{REGISTRO DE ÓBITOS}

A Tabela 1 mostra o número de óbitos detectados durante nossa pesquisa em Pelotas, comparados com as cifras liberadas pela Secretaria da Saúde e do Meio Ambiente do 
Estado para o mesmo município, no ano de 1982. Nota-se que, em mais de $40 \%$ dos óbitos perinatais, os atestados de óbito não foram preenchidos, ou não chegaram à Secretaria da Saúde, verificando-se um sub-registro de quase 50\% para mortes fetais e de $36 \%$ para óbitos de primeira semana.

Devido à magnitude do sub-registro de óbitos de primeira semana de vida, acrescido do de óbitos ocorridos no período pós-perinatal, o sub-registro da mortalidade infantil alcançou $24 \%$.

\section{TABELA 1}

Número de óbitos perinatais e infantis registrados pela Secretaria da Saúde e do Meio Ambiente do Estado do Rio Grande do Sul para a cidade de Pelotas em 1982, comparados com aqueles detectados pelo projeto "Estudo Longitudinal das crianças nascidas em 1982".

\begin{tabular}{lccc}
\hline Período & $\begin{array}{r}\text { Obitos } \\
\text { SSM A }\end{array}$ & $\begin{array}{c}\text { Óbitos } \\
\text { Detectados }\end{array}$ & $\begin{array}{c}\text { Percentual } \\
\text { de sub-registro }\end{array}$ \\
\hline Perinatais & 132 & 228 & $42,1 \%$ \\
$\quad$ Fetais & 59 & 113 & $47,8 \%$ \\
1ạ semana & 73 & 114 & $36,0 \%$ \\
7-365 dias & 111 & 128 & $13,3 \%$ \\
Infantis & 184 & 242 & $24,0 \%$ \\
\hline
\end{tabular}

Na Tabela 2, vemos o número oficial de óbitos perinatais para a cidade de Pelotas nos últimos 10 anos, fornecido pela Secretaria de Saúde do Estado. O número oscila entre 125 e 172 , com uma média ao redor de 150 . Como não há nenhuma razão que nos permita supor que a mortalidade perinatal da cidade aumentou bruscamente no ano de 1982, estes dados sugerem que o problema de sub-registro de óbitos perinatais vem ocorrendo há muito tempo.

\section{TABELA 2}

Número de b́bitos perinatais oficialmente registrados para a cidade de Pelotas, nos anos 1973.1982

\begin{tabular}{lccc}
\hline Ano & $\begin{array}{c}\text { Óbitos } \\
\text { Fetais }\end{array}$ & $\begin{array}{c}\text { Obitos } \\
\text { 1a semana }\end{array}$ & $\begin{array}{c}\text { Obitos } \\
\text { Perinatais }\end{array}$ \\
\hline 1973 & 66 & 59 & 125 \\
1974 & 94 & 69 & 163 \\
1975 & 92 & 80 & 172 \\
1976 & 63 & 89 & 152 \\
1977 & 53 & 64 & 117 \\
1978 & 60 & 91 & 151 \\
1979 & 60 & 90 & 150 \\
1980 & 55 & 77 & 132 \\
1981 & 75 & 87 & 162 \\
1982 & 59 & 73 & 132 \\
\hline
\end{tabular}

Para averiguar como foi possível a ocorrência de taxas tão elevadas de sub-registro de óbitos perinatais com nasci- 
mentos hospitalares, entrevistamos as funcionárias de uma grande maternidade da cidade, onde ocorreram $60 \%$ dos óbitos não registrados. Fomos informados que o sepultamento de um natimorto ou de um recém-nascido falecido nos primeiros dias, juntamente com outro cadáver, em um caixão comum, era prática relativamente freqüente, principalmente quando a família do recém-nascido era muito pobre. Procedendo assim, os funcionários tentavam aliviar a família das despesas de um funeral. Em muitos destes óbitos, os atestados de óbito haviam sido preenchidos, mas foram retidos por funcionários do hospital.

Com relação aos óbitos pós-perinatais, encontramos um sub-registro de $13,3 \%$, o que é bastante elevado para óbitos que ocorreram predominantemente em hospitais. Revisando o traje to percorrido pelo atestado de óbito desde o hospital até a Delegacia Regional de Saúde, constatamos que muitas famílias recebem o atestado no hospital, realizam o funeral, mas não encaminham este atestado ao cartório. Várias razões aqui intervêm, tais como a desinformação sobre a necessidade dessa medida, a ausência de certidão de nascimento e a falta de recursos para pagar as despesas do cartório. A esta última razão se acresce o fato de que, passados 15 dias do óbito, faz-se necessário um mandado judicial para certificar o óbito, procedimento este que implica gastos adicionais.

\section{DISCUSSÃO}

O elevado sub-registro de óbitos perinatais e pós-perinatais detectado nesta investigação é surpreendente, considerando que o Rio Grande do Sul é tido como um dos líderes no registro de eventos vitais no país, e que as estatísticas referem-se a óbitos ocorridos predominantemente em hospitais. A ocorrência de sub-registro de mais de $40 \%$ para mortes perinatais, quase $50 \%$ para óbitos fetais, e $24 \%$ para mortalidade infantil, leva-nos à conclusão de que as estatísticas oficiais devem ser encaradas com reserva.

Cabe ressaltar que estamos comparando a mortalidade de uma coorte com a mortalidade oficial para um ano, e, portanto, alguns óbitos ocorridos na coorte de 1982 serão incluídos na mortalidade de 1983. Entretanto, como o número oficial de óbitos para Pelotas, em 1983, foi menor do que para 1982 (164 contra 184), este tipo de problema não pode ser responsabilizado pela estimativa de sub-registro.

Uma grave conseqüência do sub-registro é a queda artificial dos coeficientes de mortalidade fetal, perinatal, neonatal e infantil, levando a uma falsa impressão de que as condições de saúde da comunidade são satisfatórias. A partir desta falsa constatação, os mais diversos usos podem ser feitos desses indicadores de saúde, desde afirmações do acerto 
de medidas de natureza política, até os relacionados com o planejamento de saúde. Com respeito a estes últimos, é óbvio que menos recursos serão destinados a um setor que demonstra uma atuação satisfatória, medida através de indicadores "confiáveis".

O fato de quase $8 \%$ dos nascimentos hospitalares não serem do conhecimento das autoridades do setor de saúde pode ter como uma de suas conseqüências o planejamento equivocado da necessidade de leitos nas matemidades e berçários, incluindo incubadoras. Além disso, os coeficientes de mortalidade calculados com um falso denominador, menor do que o real, serão superestimados.

Neste sentido, o sub-registro de óbitos seria atenuado, mas este efeito em Pelotas seria muito discreto, visto que o sub-registro de óbitos infantis foi de $24 \%$, enquanto que o sub-registro de nascimentos não alcançou $5 \%$.

A estimativa exagerada de nascimentos domiciliares também pode levar a equívocos no planejamento de saúde. Um exemplo seria o gasto desnecessário de tempo e recursos com o treinamento de parteiras para o atendimento domiciliar.

Uma razão para o descaso com relação à coleta de informações rotineiras é que a equipe de saúde, principalmente os médicos, não se julga responsável (e realmente não é responsabilizada) por esta função. Assim, quando erros ou omissões ocorrem, as pessoas diretamente envolvidas não são chamadas pelas autoridades competentes para discutir as possíveis causas do erro e receber as devidas instruções. Parece, também, que a Secretaria da Saúde envia poucas estatísticas perinatais e infantis de interesse prático para as unidades hospitalares, baseadas nas informações rotineiramente enviadas. Como não são vistas as vantagens desta tarefa, uma vez que o desempenho das unidades nunca é discutido com base nas informações coletadas, a equipe tornase desinteressada sobre a qualidade destas informações.

Ainda com relação ao sub-registro de eventos vitais, outro importante motivo para a sua existência é a distância e a falta de contato existentes entre os hospitais e os cartórios de registro. Para registrar nascimentos e óbitos, as famílias são obrigadas, após a alta hospitalar, a procurarem o cartório, onde uma taxa terá de ser paga para que se proceda o registro. Se este for feito 15 dias ou mais após o evento, às despesas habituais será acrescida uma multa para que o registro seja realizado. Em Pelotas, um problema adicional é o fato de os cartórios terem dividido entre si os registros dos hospitais da cidade, de forma que determinado cartório encarrega-se de um dado hospital, mas não de outro. Desta forma, as famílias terminam por falsear as informações, afirmando que os filhos nasceram em casa, para poderem fazer 
o registro no cartório que mais lhes convém. Isto ocorre freqüentemente com famílias residentes em áreas rurais, que preferem registrar os filhos nessas áreas e não na cidade.

As seguintes sugestões são oferecidas, com o objetivo de resolver os problemas citados:

a) criação de postos dos cartórios de registro dentro dos hospitais, para o registro obrigatório de nascimentos e óbitos antes da alta hospitalar. Esta seria uma forma fácil de desburocratizar o processo e evitar os problemas de sub-registros e falsas informações. Esse órgão funcionaria em estreito contato com o serviço de registros do hospital, e não cobraria as custas para famílias comprovadamente carentes (por exemplo, aquelas hospitalizadas como indigentes).

b) a Secretaria da Saúde poderia promover a conscientização da equipe de saúde a respeito da importância da utilização dos indicadores de saúde no trabalho diário. Uma forma prática de "treinamento em serviço" seria a distribuição periódica de um sumário da atuação de cada unidade, comparando-as com outras unidades da região, a exemplo do que é feito três vezes por ano na Suécia ${ }^{8}$. Neste sumário, seria dada ênfase a informações como: coeficientes de natalidade, morbidade e mortalidade, causas de óbito, incidência de baixo peso ao nascer, proporção de partos operatórios e ocupação de leitos hospitalares. Dessa forma, a equipe teria um aprendizado constante sobre a importância dessas informações para a monitorização dos serviços, além de saber em que aspectos específicos (por exemplo, incidência de cesareanas) a unidade deveria concentrar esforços para melhorar sua atuação.

Para atingir esta meta, a Secretaria necessitaria aprimorar seu sistema de coleta de informações, que atualmente não permite a conexão direta das informações de nascimento e óbito. Seria, também, necessário agilizar o sistema de análise de dados, o que não representaria grande problema, uma vez que pacotes de computação bastante flexíveis, como o SPSS $^{4}$ ou o SAS $^{9}$ são facilmente implantáveis.

Medidas a longo prazo incluem, necessariamente, uma mudança de atitude com relação à formação dos profissionais de saúde. Nossos cursos universitários enfatizam o atendimento individual, e não discutem suficientemente formas de trabalho em equipe com a finalidade de melhorar a saúde de coletividades. Como conseqüência, instrumentos de avaliação individual, como exames laboratoriais, são bem conhecidos e manejados, enquanto que formas de avaliação de saúde coletiva e de monitorização da eficácia do trabalho de equipes de saúde são pouco enfatizadas.

Espera-se que a progressiva conscientização dos profissionais de saúde possibilite uma melhoria da qualidade das informações disponíveis. 
Enquanto estas mudanças não ocorrem, recomendamos, baseados nos dados aqui apresentados, que as informações oficiais sobre saúde perinatal e infantil sejam encaradas com a devida cautela. Outros estudos epidemiológicos que utilizem fontes não-oficiais de coleta de informações fazem-se muito necessários, com a finalidade de se avaliar a qualidade dos registros em outras regiões.

\section{AGRADECIMENTOS}

Esta pesquisa foi financiada pelo International Development Research Centre (Canadá) e pela Overseas Development Administration (Reino Unido). Agradecemos à 3a Delegacia Regional de Saúde, com sede em Pelotas, e à Equipe de Informática da Delegacia da Saúde e do Meio Ambiente, em Porto Alegre, pela colaboração prestada durante todas as fases da investigação.

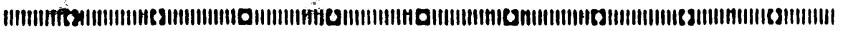

$A$ cohort of 6,011 children is being followed up from birth in Pelotas, RS, Brazil. More than $87 \%$ of these children were seen when aged 12-27 months (mean $=20$ months), when all 68,600 urban households were visited. Deaths were monitored through home visits, the review of hospital casenotes and of death certificates at the Secretariat of Health, and these findings were compared with official statistics. Approximately $42 \%$ of perinatal deaths failed to be registered, the corresponding figures being $48 \%$ for fetal and $24 \%$ for infant deaths. On the other hand, less than $5 \%$ of all births failed to be registered. The implications of these findings for health policy-makers are discussed.

\section{REFERÊNCIAS BIBLIOGRÁFICAS}

1. BARROS, F.C. The epidsmiology of perinatal health in Southern Brazil; a study of perinatal mortality, low birth weight and utilisation of health care. London, 1985. [Thesis for the degree of $\mathrm{PhD}$, University of London]

2. BARROS, F.C. et alii. Saúde perinatal em Pelotas, RS, Brasil; fatores sociais e biológicos. $R$. Saúde públ., 18: 301-12, 1984.

3. MAHNER, J. Birth weight as a new development indicator. In: STERKY, G. \& MELLANDER, L. eds. SAREC Report; birth weight distribution and indicator of social development. Uppsala, Swedish Agency for Research Cooperation with Developing Countries, 1979, p. 33-9. 
4. NIE, N.H. et alii. Statistical package for the social sciences. 2.ed. New York, McGraw-Hill, 1975.

5. RIO GRANDE DO SUL. Secretaria da Saúde e do Meio Ambiente. Saúde no Rio Grande do Sul 1970/1982. Porto Alegre, 1983.

6. RIO GRANDE DO SUL. Secretaria da Saúde e do Meio Ambiente. Sub-registro de nascimentos e óbitos no Rio Grande do Sul em 1974. Porto Alegre, 1976.

7. ROMENSKY, A.A. \& INGNAT'EVA, R.K. Certificate of cause of perinatal death, Geneva, WHO, 1975.

8. ROOTH, G. Better perinatal health; Sweden. Lancet, 2: 1170-2, 1979.

9. SAS user's guide; basics. North Carolina, SAS Institute Inc., 1982.

10. VICTORIA, C.G. et alii. Estudo longitudinal das crianças nascidas em 1982 em Pelotas, RS; metodologia e resultados preliminares. R. Saúde públ., 19:59-68, 1985. 